\title{
UNIFIED TREATMENT OF FRACTIONAL INTEGRAL INEQUALITIES VIA LINEAR FUNCTIONALS
}

\author{
M. BOMBARDELLI, L. NIKOLOVA, AND S. VAROŠANEC
}

Received 30 November, 2015

\begin{abstract}
In the paper we prove several inequalities involving two isotonic linear functionals. We consider inequalities for functions with variable bounds, for Lipschitz and Hölder type functions etc. These results give us an elegant method for obtaining a number of inequalities for various kinds of fractional integral operators such as for the Riemann-Liouville fractional integral operator, the Hadamard fractional integral operator, fractional hyperqeometric integral and corresponding q-integrals.
\end{abstract}

2010 Mathematics Subject Classification: 26D10;26A33

Keywords: Chebyshev inequality, Chebyshev difference, fractional integral operator, isotonic linear functional, Lipschitz function

\section{INTRODUCTION}

Recently several papers involving inequalities for fractional integral operators have been published, see $[3,4,7,8,19,21,22]$ and references therein. Certain similarity of those inequalities shows that those results have a common origin. In this paper we give a unified treatment of several known inequalities for fractional integral operators via theory of isotonic linear functionals. In fact, we prove general inequalities involving isotonic linear functionals from which some interesting results are followed.

The paper is organized in the following way. The rest of this section contains definitions and some examples of isotonic linear functionals connected with fractional integration and integration on time scales. The Chebyshev inequality for one and two isotonic functionals are given. Inequalities for Lipschitz functions are given in the second section. The third section is devoted to new inequalities involving two isotonic functionals and functions with variable upper and lower bounds. The fourth section is devoted to results involving more than two functions. Applications or references where we can find applications in the theory of fractional operators and calculus on time scales are also given.

The research of the second author was partially supported by the Sofia University SRF under contract No 146/2015. 


\section{Isotonic linear functionals}

Definition 1 (Isotonic linear functional). Let $E$ be a non-empty set and $L$ be a class of real-valued functions on $E$ having the properties:

L1. If $f, g \in L$, then $(a f+b g) \in L$ for all $a, b \in \mathbf{R}$;

L2. The function 1 belongs to $L$. $(\mathbf{1}(t)=1$ for $t \in E)$.

A functional $A: L \rightarrow \mathbf{R}$ is called an isotonic linear functional if

A1. $A(a f+b g)=a A(f)+b A(g)$ for $f, g \in L, a, b \in \mathbf{R}$;

A2. $f \in L, f(t) \geq 0$ on $E$ implies $A(f) \geq 0$.

There exist a lot of interesting examples of linear functionals which play some role in different parts of mathematics. In the following example we describe the most mentioned functionals - discrete and integral - , and we give several functionals which appear in the theory of fractional calculus and calculus on time scales.

Example 1. (i) Discrete functional. If $E=\{1,2, \ldots, n\}$ and $f: E \rightarrow \mathbf{R}$, then $A(f)=\sum_{i=1}^{n} f(i)$ is an isotonic linear functional.

(ii) Integral functional. If $E=[a, b] \subset \mathbf{R}$ and $L=L(a, b)$, then $A(f)=$ $\int_{a}^{b} f(t) d t$ is an isotonic linear functional. If $A_{1}(f)=\frac{1}{b-a} A(f)$, then $A_{1}$ is a normalized isotonic linear functional.

(iii) Fractional hypergeometric operator. If $t>0, \alpha>\max \{0,-\beta-\mu\}$, $\mu>-1, \beta-1<\eta<0$, then

$$
A(f)=I_{t}^{\alpha, \beta, \eta, \mu}\{f(t)\}
$$

is an isotonic linear functional, ([3]), where $I_{t}^{\alpha, \beta, \eta, \mu}\{f(t)\}$ is a fractional hypergeometric operator defined as

$$
\begin{aligned}
& I_{t}^{\alpha, \beta, \eta, \mu}\{f(t)\} \\
& \quad=\frac{t^{-\alpha-\beta-2 \mu}}{\Gamma(\alpha)} \int_{0}^{t} \sigma^{\mu}(t-\sigma)^{\alpha-1}{ }_{2} F_{1}\left(\alpha+\beta+\mu,-\eta, \alpha ; 1-\frac{\sigma}{t}\right) f(\sigma) d \sigma
\end{aligned}
$$

where the function ${ }_{2} F_{1}(a, b, c, t)=\sum_{n=0}^{\infty} \frac{(a)_{n}(b)_{n}}{(c)_{n}} \frac{t^{n}}{n !}$ is the Gaussian hypergeometric function and $(a)_{n}$ is the Pochhammer symbol:

$(a)_{n}=a(a+1) \ldots(a+n-1),(a)_{0}=1$.

- Putting $\mu=0$, then the fractional hypergeometric operator reduces to the Saigo fractional integral operator $I^{\alpha, \beta, \eta}\{f(t)\}$.

- The Erdélyi-Kober fractional integral operator $I^{\alpha, \eta}\{f(t)\}$ is a particular case of $I_{t}^{\alpha, \beta, \eta, \mu}\{f(t)\}$ when $\beta=\mu=0$. 
- One of the earliest defined and the most investigated fractional integral operator is the so-called Riemann-Liouville operator defined as

$$
J^{\alpha} f(t)=I_{t}^{\alpha,-\alpha, 0,0}\{f(t)\}=\frac{1}{\Gamma(\alpha)} \int_{0}^{t}(t-\sigma)^{\alpha-1} f(\sigma) d \sigma, \alpha>0,
$$

and it is a particular case of a fractional hypergeometric operator for $\beta=-\alpha$, $\eta=\mu=0$.

(iv) q-analogues. The above-mentioned operators have the so-called $q$-analogues. We describe a $q$-analogue of Saigo's fractional integral, [2]. Let $\Re(\alpha)>0, \beta, \eta \in \mathbf{C}$, $0<q<1$. A $q$-analogue of Saigo's fractional integral $I_{q}^{\alpha, \beta, \eta}$ is given for $|\tau / t|<1$ by equation

$$
\begin{aligned}
& I_{q}^{\alpha, \beta, \eta}\{f(t)\}=\frac{t^{-\beta-1}}{\Gamma_{q}(\alpha)} \int_{0}^{t}\left(q \frac{\tau}{t} ; q\right)_{\alpha-1} \\
& \times \sum_{m=0}^{\infty} \frac{\left(q^{\alpha+\beta} ; q\right)_{m}\left(q^{-\eta} ; q\right)_{m}}{\left(q^{\alpha} ; q\right)_{m}(q ; q)_{m}} \cdot q^{(\eta-\beta) m}(-1)^{m} q^{-m(m-1) / 2}\left(\frac{\tau}{t}-1\right)_{q}^{m} f(\tau) d_{q} \tau,
\end{aligned}
$$

where

$$
(a ; q)_{\alpha}=\frac{\prod_{k=0}^{\infty}\left(1-a q^{k}\right)}{\prod_{k=0}^{\infty}\left(1-a q^{\alpha+k}\right)} \text { and }(t-a)_{q}^{n}=t^{n}\left(\frac{a}{t} ; q\right)_{n} .
$$

If $\alpha>0, \beta, \eta \in \mathbf{R}$ with $\alpha+\beta>0$ and $\eta<0$, then $I_{q}^{\alpha, \beta, \eta}$ is isotonic, [9].

(v) The Hadamard fractional integral. The Hadamard fractional integral of order $\alpha>0$ of function $f$ is defined as

$$
{ }_{H} J^{\alpha} f(x)=\frac{1}{\Gamma(\alpha)} \int_{1}^{x}\left(\log \frac{x}{y}\right)^{\alpha-1} \frac{f(y) d y}{y}, 1<x .
$$

For further reading about fractional calculus we recommend, for example, [14].

(vi) In $1988 \mathrm{~S}$. Hilger introduced the calculus on time scales, a strong tool for unified treatment of differential and difference equations. Among different kinds of integrals the most investigated is $\Delta$-integral, see for example, $[1,15]$. A $\Delta$-integral was followed by $\nabla$-integral, $\diamond_{\alpha}$ integral, $\alpha, \beta$-symmetric integral etc. All of them are isotonic linear functionals.

\section{Chebyshev-type inequalities for isotonic linear functionals}

After that short text about various kinds of isotonic linear functionals, let us say few words about some inequalities of Chebyshev type involving isotonic linear functionals.

We say that functions $f$ and $g$ on $E$ are similarly ordered (or synchronous) if for each $x, y \in E$

$$
(f(x)-f(y))(g(x)-g(y)) \geq 0 .
$$


If the reversed inequality holds, then we say that $f$ and $g$ are oppositely ordered or asynchronous. The most famous inequality which involve similarly or oppositely ordered functions is the Chebyshev inequality for integrals. It states that if $p, f$ and $g$ are integrable real functions on $[a, b] \subset \mathbf{R}$ and if $f$ and $g$ are similarly ordered, then

$$
\int_{a}^{b} p(x) d x \int_{a}^{b} p(x) f(x) g(x) d x \geq \int_{a}^{b} p(x) f(x) d x \int_{a}^{b} p(x) g(x) d x .
$$

If $f$ and $g$ are oppositely ordered then the reverse of the inequality in (1.2) is valid.

During the last century a lot of results about the Chebyshev inequality appear. Here, we only give the most recent results involving two isotonic linear functionals, [16].

Theorem 1 (The Chebyshev inequality for two functionals). Let $A$ and $B$ be two isotonic linear functionals on $L$ and let $p, q \in L$ be non-negative functions. Let $f, g$ be two functions on $E$ such that $p f, p g, q f, q g, p f g, q f g \in L$.

If $f$ and $g$ are similarly ordered functions, then

$$
A(p f g) B(q)+A(p) B(q f g) \geq A(p f) B(q g)+A(p g) B(q f) .
$$

If $f$ and $g$ are oppositely ordered functions, then the reverse inequality in (1.3) holds.

Putting $A=B, p=q$ in (1.3) and divided by 2 we get that for similarly ordered functions $f$ and $g$ such that $p f, p g, p f g \in L$, the following holds

$$
A(p) A(p f g) \geq A(p f) A(p g) .
$$

If $f$ and $g$ are oppositely ordered functions, then the reverse inequality holds.

It is, in fact, the Chebyshev inequality for one isotonic positive functional.

One of the most investigated question related to the Chebyshev integral inequality is the question of finding bounds for the so-called Chebyshev difference which is defined as a difference between two sides from inequality (1.2). Results related to that question are called Gruüss type inequalities. In [16] Grüss type inequalities for the Chebyshev difference $T(A, B, p, q, f, g)$ which arise from inequality (1.3) are given where

$$
T(A, B, p, q, f, g)=B(q) A(p f g)+A(p) B(q f g)-A(p f) B(q g)-A(p g) B(q f) \text {. }
$$

\section{INEQUALITIES FOR $M-g$-LIPSCHITZ AND HÖLDER-TYPE FUNCTIONS}

In this section $M-g$-Lipschitz functions are considered. We say that $f$ is an $M-g-$ Lipschitz function if

$$
|f(x)-f(y)| \leq M|g(x)-g(y)|
$$

for all $x, y \in E$. If $g=i d$, then $f$ is simple called an $M$-Lipschitz function.

In the following theorem we consider two functions $f$ and $g$ which are $h_{1}-$ and $h_{2}$-Lipschitz functions with constants $M_{1}$ and $M_{2}$ respectively. 
Theorem 2. Let $A$ and $B$ be isotonic linear functionals on $L$ and let $p, q$ be nonnegative functions from $L$. Let $M_{1}, M_{2}$ be real numbers and let $f, g, h_{1}, h_{2}$ be functions such that $f$ is $M_{1}-h_{1}-$ Lipschitz and $g$ is $M_{2}-h_{2}-$ Lipschitz, i.e. for all $x, y \in E$

$$
\begin{aligned}
|f(x)-f(y)| & \leq M_{1}\left|h_{1}(x)-h_{1}(y)\right|, \\
|g(x)-g(y)| & \leq M_{2}\left|h_{2}(x)-h_{2}(y)\right| .
\end{aligned}
$$

If all the terms in the below inequality exist and $h_{1}$ and $h_{2}$ are or similarly ordered, or oppositely ordered, then

$$
|T(A, B, p, q, f, g)| \leq M_{1} M_{2} T\left(A, B, p, q, h_{1}, h_{2}\right) .
$$

Proof. Let $h_{1}$ and $h_{2}$ be similarly ordered. Multiplying the inequalities (2.1) and (2.2) we get

$$
|(f(x)-f(y))(g(x)-g(y))| \leq M_{1} M_{2}\left(h_{1}(x)-h_{1}(y)\right)\left(h_{2}(x)-h_{2}(y)\right) .
$$

It means that

$$
\begin{aligned}
& (f(x)-f(y))(g(x)-g(y)) \leq M_{1} M_{2}\left(h_{1}(x)-h_{1}(y)\right)\left(h_{2}(x)-h_{2}(y)\right) \quad \text { and } \\
& (f(x)-f(y))(g(x)-g(y)) \geq-M_{1} M_{2}\left(h_{1}(x)-h_{1}(y)\right)\left(h_{2}(x)-h_{2}(y)\right) .
\end{aligned}
$$

Since

$$
(f(x)-f(y))(g(x)-g(y))=f(x) g(x)+f(y) g(y)-f(x) g(y)-f(y) g(x)
$$

multiplying with $p(x) q(y)$ and acting on the first inequality by functional $A$ with respect to $x$ and then by functional $B$ with respect to $y$ we get

$$
\begin{aligned}
& A(p f g) B(q)+A(p) B(q f h)-A(p f) B(q g)-A(p g) B(q f) \\
& \leq M_{1} M_{2}\left(A\left(p h_{1} h_{2}\right) B(q)+A(p) B\left(q h_{1} h_{2}\right)-A\left(p h_{1}\right) B\left(q h_{2}\right)-A\left(p h_{2}\right) B\left(q h_{1}\right),\right. \\
& T(A, B, p, q, f, g) \leq M_{1} M_{2} T\left(A, B, p, q, h_{1}, h_{2}\right) .
\end{aligned}
$$

Similarly, from the second inequality we obtain

$$
T(A, B, p, q, f, g) \geq-M_{1} M_{2} T\left(A, B, p, q, h_{1}, h_{2}\right)
$$

and we get the claimed result. The case when $h_{1}$ and $h_{2}$ are oppositely ordered is proven similary.

Theorem 3. Let $A$ and $B$ be isotonic linear functionals on $L$ and let $p, q$ be nonnegative functions from $L$. Let $M$ be real number and let $f, g$ be functions such that

$$
|f(x)-f(y)| \leq M|g(x)-g(y)|, \quad \forall x, y .
$$

If all the terms in the below inequality exist, then

$$
\begin{aligned}
|A(p f g) B(q)+A(p) B(q f h)-A(p f) B(q g)-A(p g) B(q f)| & \\
& \leq M\left(A\left(p g^{2}\right) B(q)-2 A(p g) B(q g)+A(p) B\left(q g^{2}\right)\right) .
\end{aligned}
$$


Proof. Since $f$ is an $M-g$-Lipschitz function and $g$ is $1-g$-Lipschitz the desired inequality is a simple consequence of Theorem 2.

In the following table we give a list of papers where particular cases of Theorems from this section can be found.

TABLE 1. Known applications in particular cases of fractional integral operators

\begin{tabular}{c||c|c} 
Particular cases & Theorem 2 & Theorem 3 \\
\hline \hline $\begin{array}{c}\text { Riem.-Liouv. } \\
\text { oper. }\end{array}$ & $A=B, p \neq q, h_{1}=h_{2}=i d$ & {$[11$, Thm 3.5, Thm 3.7], } \\
$p \neq q, A=B$ and $A \neq B$, \\
\hline Saigo oper. & {$[23$, Thm 2.20$]$} & {$[23$, Thm 2.19$]$} \\
& $A \neq B, p \neq q, h_{1}=h_{2}=i d$ & $A \neq B, p \neq q$ \\
\hline$q$-Saigo oper. & {$[23$, Thm 3.17$]$} & {$[23$, Thm 3.16$]$} \\
& $A \neq B, p \neq q, h_{1}=h_{2}=i d$ & $A \neq B, p \neq q$, \\
\hline$q$-R-L. & {$[6$, Thm 3.4] } & {$[6$, Thm 3.3] } \\
int. operator & $A \neq B, p \neq q, h_{1}=h_{2}=i d$, & $A \neq B, p \neq q$, \\
& {$[6$, Thm 3.5$]$} & \\
\hline Riemann & $A=B, p \neq q, h_{1}=h_{2}=i d$ & \\
int. & {$[13$, Thm 2.1$], h_{1}=h_{2}=i d$} & {$[13$, Thm 4.1], } \\
time scale & $A=B, p=q=1$ & $A=B, p=q=\mathbf{1}$ \\
$\diamond_{\alpha}$ integral & $A=B, p=q, h_{1}=h_{2}=i d$ & {$[5$, Thm 5.1], } \\
\hline \multicolumn{2}{c}{$[5$, Thm 4.1$]}$, & $A=B, p=q$ \\
\hline
\end{tabular}

Let us say few words how to read the above table. In the first column we write a list of isotonic linear functionals. In the corresponding row we give a reference where applications of our Theorem 2 and Theorem 3 occur. For example, Theorem 2 for two Saigo operators, but with functions $h_{1}$ and $h_{2}$ equal to identity $i d$ can be find in paper [23] as Theorem 2.20 etc.

As we can see, our results enable us to give analogue results for other cases of linear functionals such as for fractional hypergeometric operators, for the Hadamard operators, for other kinds of integrals on time scales etc. Also, we improve existing results by using two different, more general functions $h_{1}$ and $h_{2}$ instead of identity function $i d$. For example, here we give a result for the Hadamard integral operators. Let $p=q=1, h_{1}=h_{2}=i d, \alpha, \beta>0$. If functions $f, g$ are $M_{1}-, M_{2}-$ Lipschitz respectively, then the following inequality holds

$$
\begin{aligned}
& \frac{\log ^{\beta} t}{\Gamma(\beta+1)} H^{\alpha}(f g(t))+\frac{\log ^{\alpha} t}{\Gamma(\alpha+1)}{ }_{H} J^{\beta}(f g(t)) \\
& \quad-{ }_{H} J^{\alpha}(f(t)){ }_{H} J^{\beta}(g(t))-{ }_{H} J^{\alpha}(g(t)){ }_{H} J^{\beta}(f(t))
\end{aligned}
$$




$$
\begin{aligned}
\leq & M_{1} M_{2} \frac{t^{2}}{\Gamma(\alpha) \Gamma(\beta)} \\
& \times\left[\frac{\log ^{\alpha} t \cdot \gamma(\beta, 2 \log t)}{2^{\beta} \alpha}+\frac{\log ^{\beta} t \cdot \gamma(\alpha, 2 \log t)}{2^{\alpha} \beta}-2 \gamma(\alpha, \log t) \gamma(\beta, \log t)\right]
\end{aligned}
$$

where $\gamma(s, x)$ is incomplete Gamma function, i.e. $\gamma(s, x)=\int_{0}^{x} t^{s-1} e^{-t} d t$.

The procedure when we firstly apply on some function $F(x, y)$ functional $A$ with respect to variable $x$ and then apply functional $B$ with respect to variable $y$ occurs very often in this paper. So, we use the following notation: if $F(x, y)$ is a function, then the number which appears after the above-described procedure is written as

$$
B_{y} A_{x}(F(x, y)) \text { or } B_{y} A_{x}(F) \text {. }
$$

It is worthless to say that if $A$ and $B$ are isotonic linear functionals, then a functional

$$
F \mapsto B_{y} A_{x}(F)
$$

is also an isotonic linear functional.

Theorem 4. Let $A$ and $B$ be isotonic linear functionals on $L$ and let $p, q$ be non-negative functions from $L$. If $f$ is of $r$-Hölder-type and $g$ is of $s$-Hölder-type, i.e.

$$
|f(x)-f(y)| \leq H_{1}|x-y|^{r}, \quad|g(x)-g(y)| \leq H_{2}|x-y|^{s}
$$

for all $x, y \in E$, where $H_{1}, H_{2}>0, r, s \in(0,1]$ fixed, then

$$
|T(A, B, p, q, f, g)| \leq H_{1} H_{2} \cdot B_{y} A_{x}\left(p(x) q(y)|x-y|^{r+s}\right) .
$$

Proof. It is proved in similar manner as Theorem 2.

In particular case, when $p=q=\mathbf{1}, A(f)=B(f)=\int_{a}^{b} f(x) d x$, a factor $B_{y} A_{x}\left(p(x) q(y)|x-y|^{r+s}\right)$ was calculated in [13] and it is equal to

$$
\frac{2(b-a)^{r+s+2}}{(r+s+1)(r+s+2)} \text {. }
$$

\section{INEQUALITIES FOR FUNCTIONS WITH VARIABLE BOUNDS}

In this section we collect different results for functions with variable and constant bounds. For example, if we look at the paper [19], their Theorem 4 can be seen as a particular case of the following theorem.

Theorem 5. Let $A$ and $B$ be isotonic linear functionals on $L, p, q$ be non-negative functions from L. Let $f, \phi_{1}, \phi_{2}$, be functions such that

$$
\phi_{1}(t) \leq f(t) \leq \phi_{2}(t)
$$

and all terms in the below inequality exist. Then

$$
A\left(p \phi_{2}\right) B(q f)+A(p f) B\left(q \phi_{1}\right) \geq A\left(p \phi_{2}\right) B\left(q \phi_{1}\right)+A(p f) B(q f) .
$$


Proof. We consider the inequality

$$
\left(\phi_{2}(x)-f(x)\right)\left(f(y)-\phi_{1}(y)\right) \geq 0 .
$$

It is equivalent to the following

$$
\phi_{2}(x) f(y)+f(x) \phi_{1}(y) \geq \phi_{1}(y) \phi_{2}(x)+f(x) f(y) .
$$

After multiplying with $p(x) q(y)$ and acting on this inequality first by functional $A$ with respect to $x$ and then by $B$ with respect to $y$ we get the wanted inequality.

If functions $\phi_{1}, \phi_{2}$ become constant functions, then we get the following corollary.

Corollary 1. Let $A$ and $B$ be isotonic linear functionals on $L$ and let $p, q$ be non-negative functions from $L$. Let $m, M$ be real numbers and let $f$ be function such that

$$
m \leq f(t) \leq M
$$

and all terms in the below inequality exist. Then

$$
M A(p) B(q f)+m A(p f) B(q) \geq M m A(p) B(q)+A(p f) B(q f) .
$$

Proof. Follows from Theorem 5 for $\phi_{1}(t)=m, \phi_{2}(t)=M$.

Corollary 2. Let $A$ and $B$ be isotonic linear functionals on $L$ and let $p, q$ be non-negative functions from $L$. Let $M>0$ and let $\varphi, f$ be function such that

$$
|f(t)-\varphi(t)|<M
$$

and all terms in the below inequality exist. Then

$$
\begin{gathered}
A(p \varphi) B(q f)+A(p f) B(q \varphi)+M A(p) B(q f)+M A(p \varphi) B(q)+M^{2} A(p) B(q) \\
\geq A(p \varphi) B(q \varphi)+M A(p) B(q \varphi)+M A(p f) B(q)+A(p f) B(q f) .
\end{gathered}
$$

Proof. Follows from Theorem 5 for $\phi_{1}(t)=\varphi(t)-M, \phi_{2}(t)=\varphi(t)+M$.

Theorem 6. Let $A$ and $B$ be isotonic linear functionals on $L$ and let $p, q$ be nonnegative functions from $L$. Let $\phi_{1}, \phi_{2}, \psi_{1}, \psi_{2}, f$ and $g$ be functions such that all terms in the below inequality exist and conditions

$$
\phi_{1}(t) \leq f(t) \leq \phi_{2}(t) \quad \text { and } \quad \psi_{1}(t) \leq g(t) \leq \psi_{2}(t)
$$

hold. Then

$$
\begin{aligned}
& A\left(p \phi_{1}\right) B\left(q \psi_{1}\right)+A(p f) B(q g) \geq A\left(p \phi_{1}\right) B(q g)+A(p f) B\left(q \psi_{1}\right), \\
& A\left(p \phi_{1}\right) B\left(q \psi_{2}\right)+A(p f) B(q g) \leq A\left(p \phi_{1}\right) B(q g)+A(p f) B\left(q \psi_{2}\right), \\
& A\left(p \phi_{2}\right) B\left(q \psi_{1}\right)+A(p f) B(q g) \leq A\left(p \phi_{2}\right) B(q g)+A(p f) B\left(q \psi_{1}\right), \\
& A\left(p \phi_{2}\right) B\left(q \psi_{2}\right)+A(p f) B(q g) \geq A\left(p \phi_{2}\right) B(q g)+A(p f) B\left(q \psi_{2}\right) .
\end{aligned}
$$


Proof. The inequality $\left(f(x)-\phi_{1}(x)\right)\left(g(y)-\psi_{1}(y)\right) \geq 0$ which can be written as

$$
\phi_{1}(x) \psi_{1}(y)+f(x) g(y) \geq \phi_{1}(x) g(y)+f(x) \psi_{1}(y)
$$

is obviously true.

After multiplying it with $p(x) q(y)$ and acting on this inequality first by functional $A$ with respect to $x$ and then by $B$ with respect to $y$, we get

$$
\begin{aligned}
B_{y} A_{x}\left(p ( x ) q ( y ) \left(\phi_{1}(x) \psi_{1}(y)+\right.\right. & f(x) g(y)) \\
& \geq B_{y} A_{x}\left(p(x) q(y)\left(\phi_{1}(x) g(y)+f(x) \psi_{1}(y)\right)\right.
\end{aligned}
$$

and applying properties of isotonic linear functionals $A$ and $B$ we obtain the first inequality. The other three inequalities are obtained in a similar way starting from

$$
\begin{aligned}
& \left(f(x)-\phi_{1}(x)\right)\left(\psi_{2}(y)-g(y)\right) \geq 0, \\
& \left(\phi_{2}(x)-f(x)\right)\left(g(y)-\psi_{1}(y)\right) \geq 0, \\
& \left(\phi_{2}(x)-f(x)\right)\left(\psi_{2}(y)-g(y)\right) \geq 0
\end{aligned}
$$

respectively.

Corollary 3. Let $A$ and $B$ be isotonic linear functionals on $L$ and let $p, q$ be non-negative functions from $L$. Let $m, M, n, N$ be real numbers and let $f$ and $g$ be functions such that

$$
m \leq f(t) \leq M \quad \text { and } \quad n \leq g(t) \leq N
$$

and all terms in the below inequalities exist. Then

$$
\begin{aligned}
& m n A(p) B(q)+A(p f) B(q g) \geq m A(p) B(q g)+n A(p f) B(q), \\
& m N A(p) B(q)+A(p f) B(q g) \leq m A(p) B(q g)+N A(p f) B(q), \\
& M n A(p) B(q)+A(p f) B(q g) \leq M A(p) B(q g)+n A(p f) B(q), \\
& M N A(p) B(q)+A(p f) B(q g) \geq M A(p) B(q g)+N A(p f) B(q) .
\end{aligned}
$$

Proof. Follows from Theorem 6 for $\phi_{1}=m, \phi_{2}=M, \psi_{1}=n, \psi_{2}=N$.

Theorem 7. Let $A$ and $B$ be isotonic linear functionals on $L$ and let $p, q$ be non-negative functions from $L$. Let $\theta_{1}$ and $\theta_{2}$ be a positive real numbers satisfying $\frac{1}{\theta_{1}}+\frac{1}{\theta_{2}}=1$. Let $f, \phi_{1}, \phi_{2}$, be functions such that

$$
\phi_{1}(t) \leq f(t) \leq \phi_{2}(t) .
$$

and all terms in the below inequality exist. Then

$$
\begin{aligned}
\frac{1}{\theta_{1}} B(q) A\left(p\left(\phi_{2}-f\right)^{\theta_{1}}\right) & +\frac{1}{\theta_{2}} A(p) B\left(q\left(f-\phi_{1}\right)^{\theta_{2}}\right)+A\left(p \phi_{2}\right) B\left(q \phi_{1}\right) \\
+ & A(p f) B(q f) \geq A\left(p \phi_{2}\right) B(q f)+A(p f) B\left(q \phi_{1}\right) .
\end{aligned}
$$


Proof. Let us mention the Young inequality which holds for non-negative $a, b$ and for positive $\theta_{1}$ and $\theta_{2}$ with property $\frac{1}{\theta_{1}}+\frac{1}{\theta_{2}}=1$ :

$$
\frac{1}{\theta_{1}} a^{\theta_{1}}+\frac{1}{\theta_{2}} b^{\theta_{2}} \geq a b .
$$

Setting in the previous inequality

$$
a=\phi_{2}(x)-f(x), \quad b=f(y)-\phi_{1}(y)
$$

we have

$$
\frac{1}{\theta_{1}}\left(\phi_{2}(x)-f(x)\right)^{\theta_{1}}+\frac{1}{\theta_{2}}\left(f(y)-\phi_{1}(y)\right)^{\theta_{2}} \geq\left(\phi_{2}(x)-f(x)\right)\left(f(y)-\phi_{1}(y)\right) .
$$

Applying usual procedure we get

$$
\begin{aligned}
B_{y} A_{x}\left(p ( x ) q ( y ) \left(\frac { 1 } { \theta _ { 1 } } \left(\phi_{2}(x)\right.\right.\right. & \left.-f(x))^{\theta_{1}}+\frac{1}{\theta_{2}}\left(f(y)-\phi_{1}(y)\right)^{\theta_{2}}\right) \\
& +B_{y} A_{x}\left(p(x) q(y)\left(\phi_{2}(x)-f(x)\right)\left(f(y)-\phi_{1}(y)\right)\right.
\end{aligned}
$$

and after applying properties of $A$ and $B$ we obtain

$\frac{1}{\theta_{1}} B(q) A\left(p\left(\phi_{2}-f\right)^{\theta_{1}}\right)+\frac{1}{\theta_{2}} A(p) B\left(q\left(f-\phi_{1}\right)^{\theta_{2}}\right) \geq A\left(p\left(\phi_{2}-f\right)\right) B\left(q\left(f-\phi_{1}\right)\right)$.

Using result from Theorem 5 we get inequality (3.1).

Corollary 4. Let $A$ and $B$ be isotonic linear functionals on $L$ and let $p, q$ be non-negative functions from $L$. Let $m, n \in \mathbf{R}$ and let $f$ be function such that $m \leq$ $f(t) \leq M$ and all terms in the below inequality exist. Then

$$
\begin{aligned}
(M+m)^{2} A(p) B(q)+A\left(p f^{2}\right) B(q) & +2 A(p f) B(q f)+A(p) B\left(q f^{2}\right) \\
& \geq 2(M+m)[A(p) B(q f)+A(p f) B(q)] .
\end{aligned}
$$

Proof. Follows from Theorem 7 for $\phi_{1}=m, \phi_{2}=M$ and $\theta_{1}=\theta_{2}=2$.

Theorem 8. Let $A$ and $B$ be isotonic linear functionals on $L$ and let $p, q$ be non-negative functions from L. Let $\theta_{1}$ and $\theta_{2}$ be a positive real numbers satisfying $\frac{1}{\theta_{1}}+\frac{1}{\theta_{2}}=1$. Let $\phi_{1}, \phi_{2}, \psi_{1}, \psi_{2}, f$ and $g$ be functions such that all terms in the below inequality exist and conditions

$$
\phi_{1}(t) \leq f(t) \leq \phi_{2}(t) \quad \text { and } \quad \psi_{1}(t) \leq g(t) \leq \psi_{2}(t)
$$

hold. Then

$\frac{1}{\theta_{1}} A\left(p\left(\phi_{2}-f\right)^{\theta_{1}}\right) B(q)+\frac{1}{\theta_{2}} A(p) B\left(q\left(\psi_{2}-g\right)^{\theta_{2}}\right) \geq A\left(p\left(\phi_{2}-f\right)\right) B\left(q\left(\psi_{2}-g\right)\right)$,

$\frac{1}{\theta_{1}} A\left(p\left(\phi_{2}-f\right)^{\theta_{1}}\right) B(q)+\frac{1}{\theta_{2}} A(p) B\left(q\left(g-\psi_{1}\right)^{\theta_{2}}\right) \geq A\left(p\left(\phi_{2}-f\right)\right) B\left(q\left(g-\psi_{1}\right)\right)$, 
$\frac{1}{\theta_{1}} A\left(p\left(f-\phi_{1}\right)^{\theta_{1}}\right) B(q)+\frac{1}{\theta_{2}} A(p) B\left(q\left(\psi_{2}-g\right)^{\theta_{2}}\right) \geq A\left(p\left(f-\phi_{1}\right)\right) B\left(q\left(\psi_{2}-g\right)\right)$,

$\frac{1}{\theta_{1}} A\left(p\left(f-\phi_{1}\right)^{\theta_{1}}\right) B(q)+\frac{1}{\theta_{2}} A(p) B\left(q\left(g-\psi_{1}\right)^{\theta_{2}}\right) \geq A\left(p\left(f-\phi_{1}\right)\right) B\left(q\left(g-\psi_{1}\right)\right)$.

Proof. Using the Young inequality for $a=\phi_{2}(x)-f(x), b=\psi_{2}(y)-g(y)$ we get

$$
\frac{1}{\theta_{1}}\left(\phi_{2}(x)-f(x)\right)^{\theta_{1}}+\frac{1}{\theta_{2}}\left(\psi_{2}(y)-g(y)\right)^{\theta_{2}} \geq\left(\phi_{2}(x)-f(x)\right)\left(\psi_{2}(y)-g(y)\right) .
$$

Multiplying both sides with $p(x) q(y)$ and acting on the inequality by functional $A$ with respect to $x$ and then by $B$ with respect to $y$, we get

$$
\begin{aligned}
\frac{1}{\theta_{1}} A\left(p\left(\phi_{2}-f\right)^{\theta_{1}}\right) B(q)+\frac{1}{\theta_{2}} A(p) B\left(q\left(\psi_{2}-g\right)^{\theta_{2}}\right) & \\
& \geq A\left(p\left(\phi_{2}-f\right)\right) B\left(q\left(\psi_{2}-g\right)\right) .
\end{aligned}
$$

The other three inequalities can be proved in the similar way.

In the following table we give a list of papers where particular cases of some Theorems from this section can be found.

TABLE 2. Known applications for particular cases of fractional integral operators

\begin{tabular}{c||c|c|c} 
Particular cases & Theorem 5 & Corollary 1 & Theorem 6 \\
\hline \hline R-L oper. & {$[21$, Thm 2], } & {$[21$, Cor 3], } & {$[21$, Thm 5], } \\
& $A \neq B, p=q=\mathbf{1}$ & $A \neq B, p=q=\mathbf{1}$ & $A \neq B, p=q=\mathbf{1}$ \\
\hline E-K oper. & {$[22$, Cor 2$]$} & & {$[22$, Cor 3] } \\
& $A \neq B, p=q=\mathbf{1}$ & & $A \neq B, p=q=\mathbf{1}$ \\
\hline Saigo oper. & {$[8$, Thm 8$]$} & {$[8$, remark 9$]$} & {$[8$, Thm 10] } \\
& $A \neq B, p=q=\mathbf{1}$ & $A \neq B, p=q=\mathbf{1}$ & $A \neq B, p=q=\mathbf{1}$ \\
& {$[22$, Thm1] } & {$[22$, Cor 4$]$} & {$[22$, Thm $]$} \\
& $A \neq B, p=q=\mathbf{1}$ & $A \neq B, p=q=\mathbf{1}$ & $A \neq B, p=q=\mathbf{1}$ \\
\hline Had. oper. & {$[19$, Thm 4$]$,} & {$[19$, Cor 5], } & {$[19$, Thm 18$]$,} \\
& $A \neq B, p=q=\mathbf{1}$ & $A \neq B, p=q=\mathbf{1}$ & $A \neq B, p=q=\mathbf{1}$ \\
\hline
\end{tabular}

Let us mention that the non-weighted versions of Theorems 7, 8 and Corollary 4 for two Hadamard operators $A={ }_{H} J^{\alpha}$ and $B={ }_{H} J^{\beta}$ can be found in paper [19].

As we can see, we did not find similar results for hypergeometric operators in literature. But, it is obvious that our results can be applied on two fractional hypergeometric operator or on $q$-analogues of those integral operators. 


\section{INEQUALITIES FOR THREE FUNCTIONS}

This section is devoted to the results involving three or more functions and in some sense it generalize the previous section.

Theorem 9. Let $A$ and $B$ be isotonic linear functionals on $L$ and let $p, q$ be non-negative functions from $L$. Let $f, g$ be similarly ordered functions and let $h$ be function with positive values. If all terms in the below inequality exist, then

$$
\begin{aligned}
& A(p f g h) B(q)+A(p f g) B(q h)+A(p h) B(q f g)+A(p) B(q f g h) \\
& \quad \geq A(p f h) B(q g)+A(p f) B(q g h)+A(p g h) B(q f)+A(p g) B(q f h) .
\end{aligned}
$$

If $f$ and $g$ are oppositely ordered, then the reversed inequality holds.

Proof. For similarly ordered functions $f, g$ we have $(f(x)-f(y))(g(x)-g(y)) \geq$ 0 , but then also

$$
(f(x)-f(y))(g(x)-g(y))(h(x)+h(y)) \geq 0 .
$$

This can be written as

$$
\begin{aligned}
& f(x) g(x) h(x)+f(x) g(x) h(y)+f(y) g(y) h(x)+f(y) g(y) h(y) \\
& \quad \geq f(x) g(y) h(x)+f(x) g(y) h(y)+f(y) g(x) h(x)+f(y) g(x) h(y) .
\end{aligned}
$$

Multiplying both sides by $p(x) q(y)$ and acting on this inequality first by functional $A$ with respect to $x$ and then by $B$ with respect to $y$ we get the desired inequality.

Remark 1. For $p=q$ from previous theorem we get

$$
\begin{aligned}
& A(p f g h) B(p)+A(p f g) B(p h)+A(p h) B(p f g)+A(p) B(p f g h) \\
& \quad \geq A(p f h) B(p g)+A(p f) B(p g h)+A(p g h) B(p f)+A(p g) B(p f h) .
\end{aligned}
$$

If also $A=B$ then

$$
A(p f g h) A(p)+A(p f g) A(p h) \geq A(p f h) A(p g)+A(p f) A(p g h)
$$

and for $h=$ const it reduces to the Chebyshev inequality.

Remark 2. Particular cases of inequality (4.1) are appeared in several papers for different kinds of linear functionals. For example, if $A$ and $B$ are Riemann-Liouville's operators, then a non-weighted inequality is given in Theorem 2.1 in paper [20]. If $A$ and $B$ are different fractional $q$-integral of the Riemann-Liouville-type, then (4.1) is given in [18, Thm 2.1] for $p=q$. Inequalities involving two $q$-analogues of Saigo's fractional integral operators which are particular cases of (4.1) are given in [2] as Theorems 5 and 6, while similar results for generalized $q$-Erdélyi-Kober fractional integral operators are given in [17, Thm 1 and 2]. 
Lemma 1. Let $A$ and $B$ be isotonic linear functionals on $L$ and let $p, q$ be nonnegative functions from $\mathrm{L}$. Let

$$
\begin{aligned}
H_{f, g, h}(x, y)= & (f(x)-f(y))(g(x)-g(y))(h(x)-h(y)) \\
= & f(x) g(x) h(x)+f(x) g(y) h(y)+f(y) g(x) h(y)+f(y) g(y) h(x) \\
& -f(y) g(x) h(x)-f(x) g(y) h(x)-f(x) g(x) h(y)-f(y) g(y) h(y) .
\end{aligned}
$$

Then

$$
\begin{aligned}
A_{x} & B_{y}\left(p(x) q(y) H_{f, g, h}(x, y)\right) \\
= & A(p f g h) B(q)+A(p f) B(q g h)+A(p g) B(q f h)+A(p h) B(q f g) \\
& -A(p g h) B(q f)-A(p f h) B(q g)-A(p f g) B(q h)-A(p) B(q f g h) .
\end{aligned}
$$

Theorem 10. Let $A$ and $B$ be isotonic linear functionals on $L$ and let $p, q$ be non-negative functions from $L$. Let $m, M, n, N, k, K$ be real numbers and let $f, g$, $h$ be functions such that

$$
m \leq f(x) \leq M, \quad n \leq g(x) \leq N, \quad k \leq h(x) \leq K, \quad \forall x .
$$

If all the terms in the below inequality exist, then

$$
\begin{aligned}
& \mid A(p f g h) B(q)+A(p f) B(q g h)+A(p g) B(q f h)+A(p h) B(q f g) \\
& \quad-A(p f g) B(q h)-A(p f h) B(q g)-A(p g h) B(q f)-A(p) B(f g h) \mid \\
& \leq(M-m)(N-n)(K-k) A(p) B(q) .
\end{aligned}
$$

Proof. From $m \leq f(x) \leq M$ it follows $|f(x)-f(y)| \leq M-m$. Therefore

$$
\begin{gathered}
|(f(x)-f(y))(g(x)-g(y))(h(x)-h(y))| \leq(M-m)(N-n)(K-k), \text { i.e. } \\
\left|H_{f, g, h}(x, y)\right| \leq(M-m)(N-n)(K-k) .
\end{gathered}
$$

Multiplying both sides by $p(x) q(y)$ and using Lemma 1 we get

$$
\begin{aligned}
& \mid A(p f g h) B(q)-A(p f g) B(q h)-A(p f h) B(q g)+A(p f) B(q g h) \\
& \quad-A(p g h) B(q f)+A(p g) B(q f h)+A(p h) B(q f g)-A(p) B(f g h) \mid \\
& \leq(M-m)(N-n)(K-k) A(p) B(q),
\end{aligned}
$$

which proves the theorem.

Remark 3. Particular cases of Theorem 10 are appeared in [2, Thm 8 and 9] for two $q$-analogues of Saigo's fractional integral operators and in [17, Thm 3 and 4] for generalized $q$-Erdélyi-Kober fractional integral operators.

Theorem 11. Let $A$ and $B$ be isotonic linear functionals on $L$ and let $p, q$ be nonnegative functions from $L$. Let $M_{1}, M_{2}, M_{3}$ be real numbers and let $f_{i},(i=1,2,3)$ be $M_{i}-g$-Lipschitz functions. If all the terms in the below inequality exist, then $\mid A\left(p f_{1} f_{2} f_{3}\right) B(q)+A\left(p f_{1}\right) B\left(q f_{2} f_{3}\right)+A\left(p f_{2}\right) B\left(q f_{1} f_{3}\right)+A\left(p f_{3}\right) B\left(q f_{1} f_{2}\right)$ 


$$
\begin{aligned}
& -A\left(p f_{1} f_{2}\right) B\left(q f_{3}\right)-A\left(p f_{1} f_{3}\right) B\left(q f_{2}\right)-A\left(p f_{2} f_{3}\right) B\left(q f_{1}\right)-A(p) B\left(q f_{1} f_{2} f_{3}\right) \mid \\
\leq & M_{1} M_{2} M_{3} \cdot B_{y} A_{x}\left(p(x) q(y)|g(x)-g(y)|^{3}\right) .
\end{aligned}
$$

Proof. If $f_{i},(i=1,2,3)$ are $M_{i}-g$-Lipschitz functions, then

$$
\begin{gathered}
\left|f_{1}(x)-f_{1}(y)\right| \leq M_{1}|g(x)-g(y)|, \quad\left|f_{2}(x)-f_{2}(y)\right| \leq M_{2}|g(x)-g(y)|, \\
\left|f_{3}(x)-f_{3}(y)\right| \leq M_{3}|g(x)-g(y)|
\end{gathered}
$$

for all $x, y$. Multiplying those inequalities we get

$$
\left|H_{f_{1}, f_{2}, f_{3}}(x, y)\right| \leq M_{1} M_{2} M_{3}|g(x)-g(y)|^{3} .
$$

This is equivalent to

$$
\begin{aligned}
H_{f_{1}, f_{2}, f_{3}}(x, y) & \leq M_{1} M_{2} M_{3}|g(x)-g(y)|^{3} \text { and } \\
-H_{f_{1}, f_{2}, f_{3}}(x, y) & \leq M_{1} M_{2} M_{3}|g(x)-g(y)|^{3} .
\end{aligned}
$$

Multiplying both inequalities with $p(x) q(y)$ and acting on the resulting inequalities by $A$ with respect to $x$ and then by $B$ with respect to $y$, we get the desired result.

Remark 4. In [2, Thm 11 and 12] and [17, Thm 5 and 6] the authors attempted to give corresponding results for $q$-analogues of Saigo's fractional integral operators and for generalized $q$-Erdélyi-Kober fractional integral operators, respectively. But they used assumptions $\left|f_{i}(x)-f_{i}(y)\right| \leq M_{i}(x-y), i=1,2,3, x, y>0$ which leads to conclusion that $f_{i} \equiv 0$.

Remark 5. Considering results from this and from the previous section it is clear how Theorems 2 and 11 can be generalized for $n M_{i}-g$-Lipschitz functions $f_{i}$, $i=2, \ldots, n$. We leave it to a reader.

Results with two functions and three weights

The following result is based on the succesive using of the Chebyshev inequality for pairs of weights.

Theorem 12. Let $A$ and $B$ be isotonic linear functionals on $L$ and let $p, q, r$ be non-negative functions from $L$. If $f$ and $g$ are similarly ordered functions, then

$$
\begin{aligned}
& A(p)[2 A(q) B(r f g)+A(r) B(q f g)+B(r) A(q f g)] \\
& \quad+A(p f g)[A(q) B(r)+A(r) B(q)] \\
& \geq A(p)[A(q f) B(r g)+A(q g) B(r f)]+A(q)[A(p f) B(r g)+A(p g) B(r f)] \\
& \quad+A(r)[A(p f) B(q g)+A(p g) B(q f)],
\end{aligned}
$$

under assumptions that all terms are well-defined.

If $f$ and $g$ are oppositely ordered functions, then the reversed inequality holds. 
Proof. Replacing in (1.3) $p$ by $q$ and $q$ by $r$ and multiplying by $A(p)$ we get

$$
A(p)[A(q) B(r f g)+B(r) A(q f g)] \geq A(p)[A(q f) B(r g)+A(q g) B(r f)] .
$$

Replacing in (1.3) $q$ by $r$ and multiplying by $A(q)$ we get

$$
A(q)[A(p) B(r f g)+B(r) A(p f g)] \geq A(q)[A(p f) B(r g)+A(p g) B(r f)] .
$$

Multiplying (1.3) by $A(r)$ we get

$$
A(r)[A(p) B(q f g)+B(q) A(p f g)] \geq A(r)[A(p f) B(q g)+A(p g) B(q f)] .
$$

Adding the above inequalities we get the statement of the theorem.

Remark 6. Theorem 12 are proved in several papers for different kinds of linear operators. For example, if $A$ and $B$ are the Riemann-Liouville operators, then it is given in [10]. Result involving Hadamard operators is given in [7], while an analogue result for the Saigo operators and $q$-analogue of Saigo's operators are given in [9] and [23].

\section{REFERENCES}

[1] R. Agarwal, M. Bohner, and A. Peterson, "Inequalities on time scales: a survey," Mathematical Inequalities and Applications, vol. 4, pp. 535-557, 2001.

[2] D. Baleanu and P. Agarwal, "Certain inequalities involving the fractional $q$-integral operators," Abstract and Applied Analysis, vol. 2014, no. Article ID 371274, 2014.

[3] D. Baleanu, S. D. Purohit, and P. Agarwal, "On fractional integral inequalities involving hypergeometric operators," Chinese Journal of Mathematics, vol. 2014, no. Article ID 609476, 2014.

[4] S. Belarbi and Z. Dahmani, "On some new fractional integral inequalities," Journal JIPAM, vol. 19, no. 3, p. Art. 86, 2009.

[5] M. Bohner, T. Mathews, and A. Tuna, "Diamond-alpha Grüss type inequalities on time scales," Int. J. Dynamical Systems and Differential Equation, vol. 3, no. 1/2, pp. 234-247, 2011.

[6] K. Brahim and S. Taf, "On some fractional q-integral inequalities," Malaya Journal of Matematik, vol. 3, no. 1, pp. 21-26, 2013.

[7] V. L. Chinchane and D. B. Pachpatte, "On some integral inequalities using Hadamard fractional integral," Malaya Journal of Matematik, vol. 1, no. 1, pp. 62-66, 2012.

[8] V. L. Chinchane and D. B. Pachpatte, "On some Grüss-type fractional inequalities using Saigo fractional integral operator," Journal of Mathematics, vol. 2014, no. Article ID 527910, 2014.

[9] J. Choi and P. Agarwal, "Some new Saigo type fractional integral inequalities and their $q$ analogues," Abstract and Applied Analysis, vol. 2014, no. Article ID 579260, 2014.

[10] Z. Dahmani, "New inequalities in fractional integrals," International Journal of Nonlinear Science, vol. 9, no. 4, pp. 493-497, 2010.

[11] Z. Dahmani, "Some results associated with fractional integrals involving the extended $C$ hebyshev functional," Acta Universitatis Apulensis, vol. 27, pp. 217-224, 2011.

[12] Z. Dahmani, L. Tabharit, and S. Taf, "Some fractional integral inequalities," J. Nonlinear Science. Lett A, vol. 1, no. 2, pp. 155-166, 2010.

[13] S. S. Dragomir, "Some integral inequalities of Grüss type," Indian J. Pure Appl. Math., vol. 31, no. 4, pp. 397-415, 2000.

[14] V. Kiryakova, Generalized Fractional Calculus and Applications, ser. Pitman Research Notes in Math. Series. New York: Longman and J. Wiley, 1994, vol. 301. 
[15] L. Nikolova and S. Varošanec, "Chebyshev-Grüss type inequalities on time scales via two linear isotonic functionals," Mathematical Inequalities and Applications, vol. 19, no. 4, pp. 1417-1427, 2016.

[16] L. Nikolova and S. Varošanec, "Chebyshev and Grüss type inequalities involving two linear functionals and applications," Mathematical Inequalities and Applications, vol. 19, no. 1, pp. 127-143, 2016.

[17] D. Ritelli and P. Agarwal, "On some new inequalities involving generalized Erdélyi-Kober fractional q-integral operator," arXiv:1405.6829v1.

[18] B. Sroysang, "A study on a new fractional integral inequality in quantum calculus," Adv. Studies Theor. Phys, vol. 7, no. 14, pp. 689-692, 2014.

[19] W. Sudsutad, S. K. Ntouyas, and J. Tariboon, "Fractional integral inequalities via Hadamard's fractional integral," Abstract and Applied Analysis, vol. 2014, no. Article ID 563096, 2014.

[20] W. T. Sulaiman, "Some new fractional integral inequalities," Journal of Mathematical Analysis, vol. 2, no. 2, pp. 23-28, 2011.

[21] J. Tariboon, S. K. Ntouyas, and W. Sudsutad, "Some new Riemann-Liouville fractional integral inequalities," International Journal of Mathematics and Mathematics Sciences, vol. 2014, no. Article ID 869434, 2014.

[22] G. Wang, H. Harsh, S. D. Purohit, and T. Gupta, "A note on Saigo's fractional integral inequalities," Turkish Journal of Analysis and Number Theory, vol. 2, no. 3, pp. 65-69, 2014.

[23] W. Yang, "Some new Chebyshev and Grüss-type integral inequalities for Saigo fractional integral operators and their $q$-analogues," Filomat, vol. 29, no. 6, pp. 1269-1289, 2015.

Authors' addresses

\section{Bombardelli}

Department of Mathematics, University of Zagreb, Zagreb, Croatia

E-mail address: bombarde@math.hr

\section{Nikolova}

Department of Mathematics and Informatics, Sofia University, Sofia, Bulgaria

E-mail address: ludmilanefmi.uni-sofia.bg

S. Varošanec

Department of Mathematics, University of Zagreb, Zagreb, Croatia

E-mail address: varosans@math.hr 